\title{
Discovery of eRASSt J192932.9-560346: A bright, two-pole accreting, eclipsing polar
}

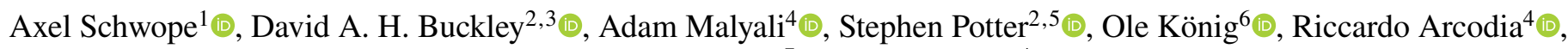 \\ Mariusz Gromadzki ${ }^{7}$, and Arne $\operatorname{Rau}^{4}{ }^{\oplus}$ \\ ${ }^{1}$ Leibniz-Institut für Astrophysik Potsdam (AIP), An der Sternwarte 16, 14482 Potsdam, Germany \\ e-mail: aschwope@aip.de \\ ${ }^{2}$ South African Astronomical Observatory, PO Box 9, Observatory, 7935 Cape Town, South Africa \\ ${ }^{3}$ Department of Astronomy, University of Cape Town, Private Bag X3, Rondebosch 7701, South Africa \\ ${ }^{4}$ Max-Planck-Institut für extraterrestrische Physik, Gießenbachstraße, 85748 Garching, Germany \\ ${ }^{5}$ Department of Physics, University of Johannesburg, PO Box 524, Auckland Park 2006, South Africa \\ ${ }^{6}$ Dr. Karl Remeis-Sternwarte \& Erlangen Centre for Astroparticle Physics, Friedrich-Alexander-Universität Erlangen-Nürnberg, \\ Sternwartstr. 7, 96049 Bamberg, Germany \\ ${ }^{7}$ Astronomical Observatory, University of Warsaw, Al. Ujazdowskie 4, 00-478 Warszawa, Poland
}

Received 28 June 2021 / Accepted 4 August 2021

\begin{abstract}
We report the discovery of a bright $(V \sim 15)$, eclipsing, two-pole accreting magnetic cataclysmic variable $(\mathrm{CV})$, a polar, as counterpart of the SRG/eROSITA and Gaia transients eRASSt J192932.9-560346 and Gaia21bxo. Frequent large-amplitude changes of its brightness at X-ray and optical wavelengths by more than four magnitudes are indicative of a CV nature of the source. Identification spectra obtained with the $10 \mathrm{~m}$ SALT telescope revealed the typical features of a magnetic CV: strong, broad HeI, HeII, and hydrogen Balmer emission lines superposed on a blue continuum. Time-resolved photoelectric polarimetry revealed that the circular polarization varies from -20 to $+20 \%$ and the linear polarization varies from 0 to $10 \%$, confirming the system to be a magnetic CV of the polar subclass. High-cadence photometry revealed deep, structured eclipses, indicating that the system is a two-pole accretor. The orbital period determined from the eclipse times is $92.5094 \pm 0.0002 \mathrm{~min}$. The X-ray spectrum is thermal only, and the implied luminosity is $L_{\mathrm{X}}=2.2 \times 10^{31} \mathrm{erg} \mathrm{s}^{-1}$ at the Gaia-determined distance of $376 \mathrm{pc}$.
\end{abstract}

Key words. stars: individual: eRASSt 192932.9-560346 - X-rays: stars - novae, cataclysmic variables

\section{Introduction}

The eROSITA instrument (Predehl et al. 2021) on board the Spektrum-Roentgen-Gamma spacecraft (SRG; Sunyaev et al. 2021) has been scanning the $X$-ray sky with an imaging telescope in the energy band between 0.2 and $10 \mathrm{keV}$ since December 2019. Eventually, it will perform eight independent all-sky surveys, called eRASSn $(n=1 \ldots 8)$, each lasting half a year. At the time of writing, eRASS3 is ongoing.

The sensitivity of eROSITA means that several thousand new compact accreting binaries are expected to be found in the final stacked version of the eROSITA all-sky survey called eRASS:8 (Merloni et al. 2012; Schwope 2012). Identifying them among the several million point-like X-ray sources detected with the sensitive instrument needs comprehensive optical identification programs that have just begun within the $\operatorname{SDSS}^{1}$ or are planned with the 4MOST facility ${ }^{2}$ (Kollmeier et al. 2017; de Jong et al. 2019). Before arrival of the stacked eROSITA catalogs, new identifications are posssible by exploiting the multidimensional parameter space spanned by time variability (at X-ray and other wavelengths), optical, infrared, and X-ray colors, location in the color-magnitude diagram, and similar.

\footnotetext{
Sloan Digital Sky Survey.

2 4-metre Multi-Object Spectroscopic Telescope.
}

Cataclysmic variables (CVs) are semidetached close compact binaries containing a Roche-lobe-filling donor star and an accreting white dwarf. While accretion in nonmagnetic CVs occurs through disks that act as mass storage, the accretion processes are much more direct in the magnetic systems that lack a disk as intermediary. This means that changes in the mass transfer rate from the donor to the white dwarf may manifest themselves instantaneously in the observed brightness of a source. Two subclasses are defined among the magnetic CVs: polars, which typically have short orbital periods $\left(P_{\text {orb }}<2\right.$ hours $)$ and similar white-dwarf rotation periods, and intermediate polars, which typically are long-period systems $\left(P_{\text {orb }}>3\right.$ hours $)$ and posses a more or less freely spinning white dwarf. The two classes are of prime interest for close binary evolution and the formation of strong magnetic fields in white dwarfs (e.g., Schreiber et al. 2021). As a group, they are relevant for the total X-ray output of our own Milky Way galaxy (Revnivtsev et al. 2009).

Here we report the discovery of a bright, eclipsing, magnetic $\mathrm{CV}$ as counterpart of a variable eRASS source. It attracted our attention by its pronounced X-ray variability in the first three allsky X-ray scans. The new eROSITA source was associated with a nearby Gaia object (offset position 2.' 6) that was reported earlier by the Gaia alert team to be a possible $\mathrm{CV}$ given its extreme photometric variability (Hodgkin et al. 2021). 

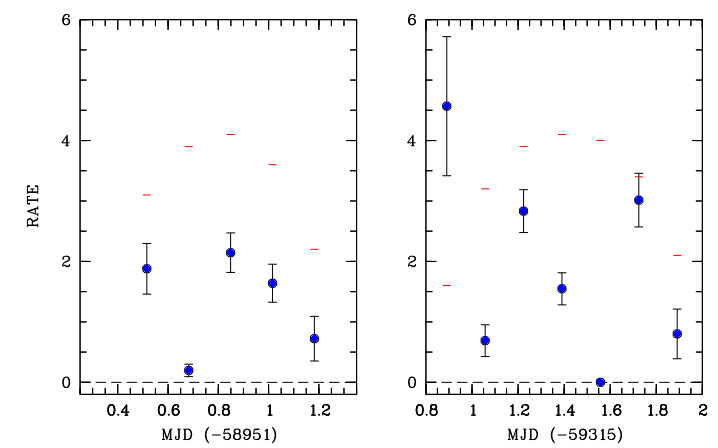

Fig. 1. Full-band eRASS1 and eRASS3 X-ray light curves $(0.2-10 \mathrm{keV})$. Shown are the vignetting-corrected mean rates per eRODAY with blue symbols. The exposure time per eRODAY is indicated with short red dashes (divided by a factor 10 ).

We describe the initial eROSITA results (Sect. 2), present the data in context with archival X-ray observations (Sect. 3), and report our SALT (Southern African Large Telescope) identification spectra together with time-resolved photometry and polarimetry obtained from SAAO (Sect. 4). Together, this uniquely identifies the source. It offers great potential for indepth studies of the accretion geometry through detailed eclipse analysis and is comparable to EXOSAT and ROSAT discoveries made some 30 years ago (see, e.g., Beuermann et al. 1988; Schwope et al. 1993, for UZ For and HU Aqr).

\section{X-ray observations with eROSITA}

During eRASS3, eROSITA redetected a source at $F_{\mathrm{X}}(0.6-$ $2.3 \mathrm{keV})=(2.6 \pm 0.4) \times 10^{-12} \mathrm{erg} \mathrm{cm}^{-2} \mathrm{~s}^{-1}$ that was undetected in eRASS2 $\left(F_{\mathrm{X}}(0.6-2.3 \mathrm{keV})<8 \times 10^{-14} \mathrm{erg} \mathrm{cm}^{-2} \mathrm{~s}^{-1}\right)$, but was previously found at $F_{\mathrm{X}}(0.6-2.3 \mathrm{keV})=(1.8 \pm 0.3) \times$ $10^{-12} \mathrm{erg} \mathrm{cm}^{-2} \mathrm{~s}^{-1}$ in eRASS1. The X-ray source position determined in eRASS3 is $\operatorname{RA}(J 2000)=19: 29: 32.9$, $\operatorname{Dec}(\mathrm{J} 2000)=-56: 03: 46$, with a statistical uncertainty of 1.3 arcsec.

The area of the transient was covered in eRASS1 between 2020-04-12 08:22:21 and 2020-04-13 04:22:41, in eRASS2 between 2020-10-14T20:59:40 and 2020-10-19 01:00:10, and in eRASS3 between 2021-04-11 21:22:14 and 2021-04-12 21:22:44 (all times in UTC). During these surveys 121 (228) and 181 (388) photons were registered with the standard source-detection pipeline in the main band between $0.6 \mathrm{keV}$ and $2.3 \mathrm{keV}$, respectively (the numbers in parentheses refer to the full band $0.2-10 \mathrm{keV}$; all numbers given were derived with pipeline version c946, see Brunner et al. 2022, for details of the detection pipeline). The original photon event tables for each sky survey were binned to give mean count rates per scan ${ }^{3}$. Light curves in the full spectral bandpass, $0.2-10 \mathrm{keV}$, are shown in Fig. 1. The mean rates in eRASS 1 and 3 were $1.32 \mathrm{~s}^{-1}$ and $1.92 \mathrm{~s}^{-1}$, and the summed exposures were $169 \mathrm{~s}$ and $223 \mathrm{~s}$. The object showed a variability of up to $100 \%$ between eRODAYs. In eRASS3, the fifth scan, centered on 2021-04-12 13:22:32 (UTC) lasting 37 seconds, revealed no source photon.

An X-ray spectral analysis of data obtained during eRASS1 and 3 was performed using the data from all seven telescope modules jointly. The pipeline-processed spectra were grouped

\footnotetext{
3 Individual scans are separated by 4 hours, the length of one eRODAY. Scans over a given celestial position may reach an exposure time of up to $40 \mathrm{~s}$ dependent on the off-axis angle.
}

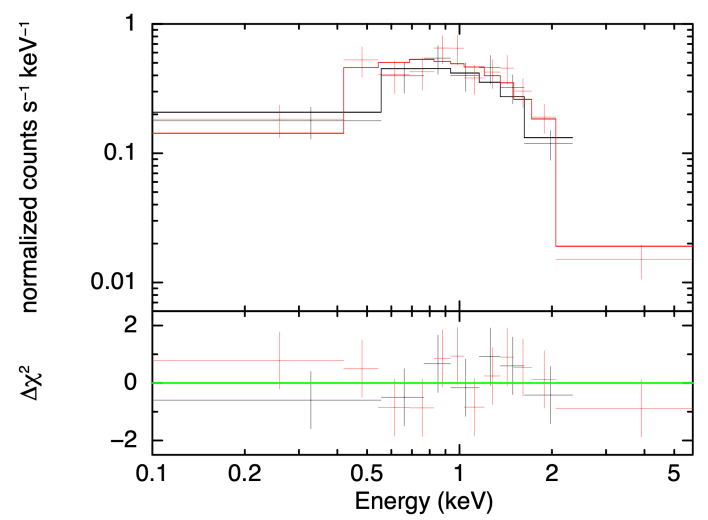

Fig. 2. eRASS1 (black) and eRASS3 (red) spectra fit with a thermal model. The lower panel shows the fit residuals normalized to their error bars.

using the HEASARC grppha command, so that each bin contained a minimum of 16 photons. Because of the $\mathrm{CV}$ nature of the source, a thermal plasma emission model was chosen initially, modified only by some cold interstellar absorbing matter (tbabs*APEC in XSPEC terminology with wilms abundances). The data were well represented by this model with $\chi^{2}=9.1$ for 15 degrees of freedom (Fig. 2). We emphasize that it was not necessary to include a soft blackbody-like spectral component to achieve a successful fit, in contrast to all the ROSAT-discovered polars. The fit does not imply strong constraints on the column density. $N_{\mathrm{H}}$ was found to be lower than $3 \times 10^{20} \mathrm{~cm}^{-2}$ (90\% confidence) and even compatible with zero. The main implication is that there is little or no intrinsic absorption in the system. The best-fit plasma temperature is about $10 \mathrm{keV}(>5 \mathrm{keV}$ at $90 \%$ confidence, not constrained toward high temperature). The bolometric flux during eRASS3 was $2.6 \times 10^{-12} \mathrm{erg} \mathrm{cm}^{-2} \mathrm{~s}^{-1}$, which gives a luminosity of $2.2 \times 10^{31} \mathrm{erg} \mathrm{s}^{-1}$ (assumed distance $376_{-14}^{+15} \mathrm{pc}$, Bailer-Jones et al. 2021).

\section{Archival X-ray and optical photometric observations}

Several previous observations of the field of the new transient were made with the Swift/XRT satellite and with XMM-Newton (slew survey, Saxton et al. 2008). In Fig. 3 all data are presented in context. The source was not discovered with XMM-Newton, that is, it was fainter than during eRASS1 or eRASS3. In the Swift/XRT data, the source was detected six times, and all data were consistent with each other at a flux of about $5 \times 10^{-13} \mathrm{erg} \mathrm{cm}^{-2} \mathrm{~s}^{-1}(0.2-2 \mathrm{keV})$. One Swift/XRT pointing revealed only a nondetection. In summary, over the past ten years, eRASSt J192932.9-560346 showed X-ray variability by a factor $>20$.

The new transient X-ray source was associated with the Gaia object 6640165468504197120 at RA $=19: 29: 33.110$, Dec $=-56: 03: 42.98,2$.' 6 away from the X-ray position. This source was announced as Gaia transient Gaia21bxo on 2021 March $14^{4}$. Gaia reported a fading and rebrightening of the source by 4 mag with a floor at about $G \simeq 20.0$ and fuzzy maximum brightness. It was labeled a CV candidate by the Gaia team. Further archival photometric observations were reported by the CRTS and ASAS-SN automated sky surveys (Drake et al. 2009; Shappee et al. 2014; Kochanek et al. 2017) and uncovered

\footnotetext{
4 http://gsaweb.ast.cam.ac.uk/alerts/alert/Gaia21bxo/, see also https: //www . wis-tns.org/object/2021kdp
} 


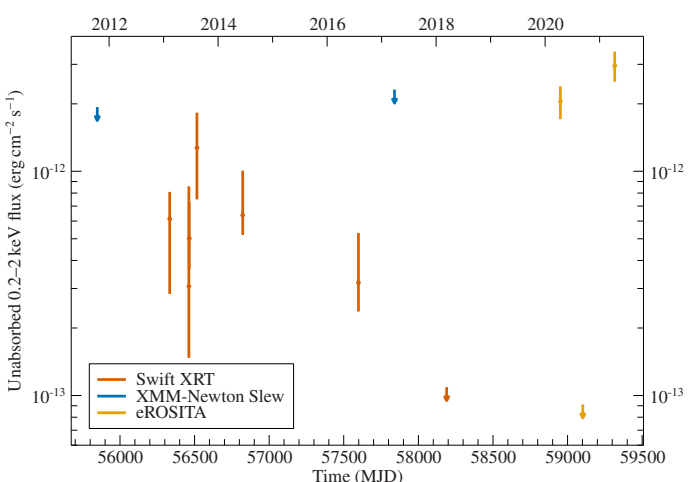

Fig. 3. Long-term X-ray light curve of eRASSt J192932.9-560346 using XMM-Newton, Swift/XRT, and eROSITA data.

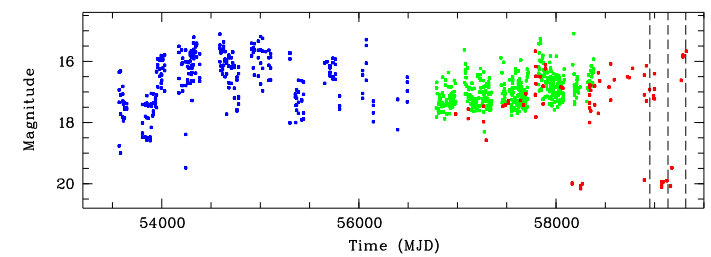

Fig. 4. CRTS (blue, white-light), ASAS-SN (green, $g$-filter), and Gaia (red, $G$ ) archival photometric data. The magnitudes are shown as published. Vertical dashed lines indicate the first three eRASS epochs.

long- and short-term variability between 15 th and 19th magnitude. The archival photometric data from CRTS, ASAS-SN, and Gaia are shown together in Fig. 4. Pre-Gaia data, in particular the CRTS, showed a high degree of optical variability on long (years) and short (days) timescales, but the source was never before observed at magnitudes as faint as $G=20$.

\section{Optical follow-up observations}

\subsection{SALT spectroscopy}

Two pairs of optical spectra, all with $300 \mathrm{~s}$ exposure, were obtained using the RSS instrument (Burgh et al. 2003) on SALT (Buckley et al. 2006), beginning on 2021 May 6, 23:53:42 UTC, and finishing at 2021 May 7, 00:17:10. The first pair of spectra covered the wavelength range 3920-6975 $\AA$, and the second pair of redder spectra covered $6035-9000 \AA$, both at a resolution of $5.5 \AA$. The blue continuum, which peaks at around $4500 \AA$, and pronounced emission lines of hydrogen and helium identify the object as a cataclysmic variable. The blue spectra are shown in Fig. 5. No significant features were seen in the red spectra, except for the $\mathrm{H} \alpha$ and $\mathrm{HeI} \lambda 6678 \AA$ emission lines.

The lines appeared rather symmetric at a blueshift of up to $600 \mathrm{~km} \mathrm{~s}^{-1}$ and with a full width at half maximum (FWHM) corresponding to about $1500 \mathrm{~km} \mathrm{~s}^{-1}$. The measured equivalent width of $\mathrm{H} \beta$ and of the ionized helium line HeII $\lambda 4686$ was up to approximately -12 and $-8 \AA$, respectively. The high flux ratio $\mathrm{HeII} / \mathrm{H} \beta \simeq 0.7$ suggests a magnetic nature (e.g., Warner 2003), which was later confirmed from polarimetry.

\subsection{HIPPO polarimetry and fast photometry}

Time-resolved photopolarimetry using the two-channel allStokes polarimeter, HIPPO (Potter et al. 2010), was conducted on

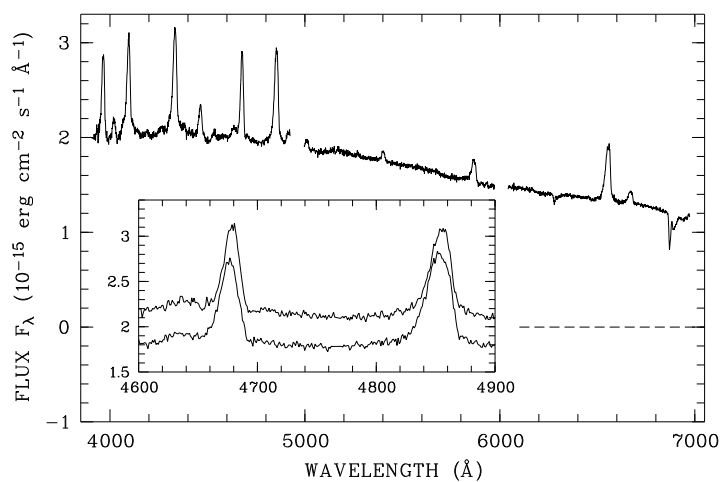

Fig. 5. SALT spectra obtained of eRASSt J192932.9-560346 on 2021 May 6. The larger trace shows the mean of the two blue exposures, and the inset shows both spectra individually zoomed on the spectral range with $\mathrm{H} \beta$ and HeII $\lambda 4686$. The dashed line in the main panel indicates zero spectral flux.
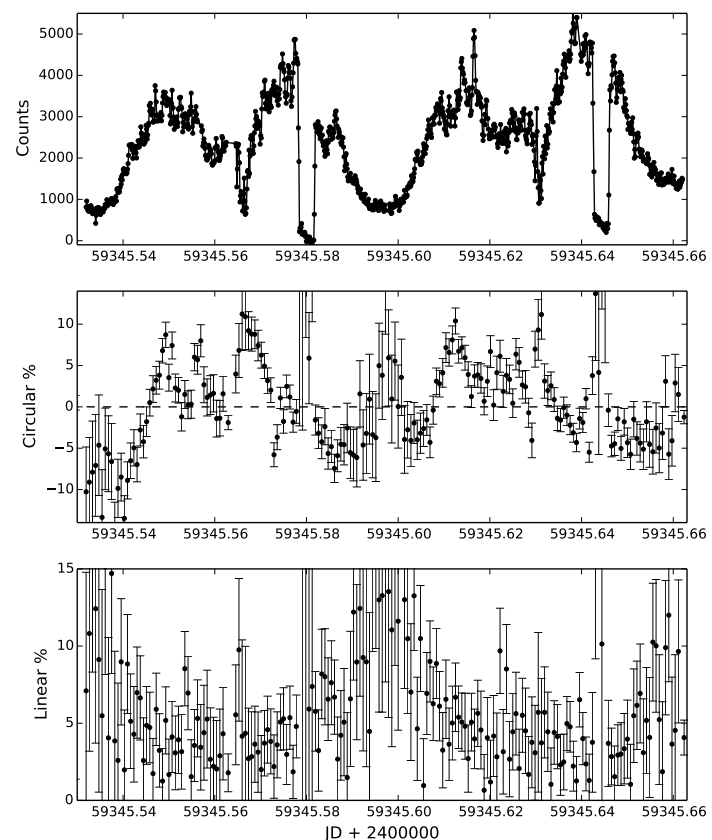

Fig. 6. HIPPO polarimetry on 2021 May 10. Upper panel: Stokes $I$ values. Middle and bottom panels: percentage of circular and linear polarizations, respectively.

the SAAO 1.9 m telescope on2021 May 9, 10, 11 and 16. Observations were taken with clear broad blueband (Schott BG39, covering $\sim 3300-6000 \AA$ ) or broad redband (Schott OG57, covering $>5700 \AA$ ) filters. The observations lasted from between 1.7 and $3.5 \mathrm{~h}$ on each night.

HIPPO is a photon-counting instrument, employing a GaAs photomultiplier with response from $\sim 3500-9000 \AA$, with an intrinsic $1 \mathrm{~ms}$ time resolution, although the polarization data are accumulated at every $0.1 \mathrm{~s}$ waveplate rotation cycle. The linear and circular polarization derived from the Stokes parameters were derived after binning the data to $60 \mathrm{~s}$ time intervals. The results for the observations on May 10 are presented in Fig. 6. The circular polarization is seen to vary from -20 to $20 \%$. It is higher in the blue. The linear polarization varies from 0 to $10 \%$. This confirms the system as a magnetic $\mathrm{CV}$ of the polar subclass. Detailed analysis and modeling of these observations will be the subject of a future paper. 

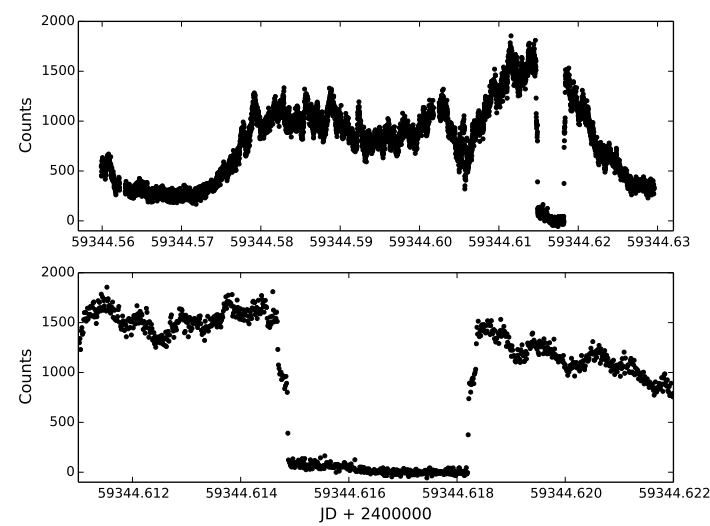

Fig. 7. Fast ( $1 \mathrm{~s})$ photometry from HIPPO observations undertaken on 2021 May 9. Details of the eclipse are shown in the lower panel, in which the ingress and egress resolve the two accretion spots.

Table 1. Optical and X-ray eclipse times of eRASStJ192932.9560346.

\begin{tabular}{rrll}
\hline \hline No & Cycle & Mid-eclipse time & Eclipse length \\
\hline 1 & 0 & $2459344.620060(17)$ & 0.003338 \\
2 & 15 & $2459345.583701(17)$ & 0.003338 \\
3 & 16 & $2459345.647945(17)$ & 0.003335 \\
4 & 30 & $2459346.547328(17)$ & 0.003333 \\
5 & 31 & $2459346.611580(17)$ & 0.003334 \\
6 & 32 & $2459346.675817(17)$ & 0.003328 \\
7 & 107 & $2459351.494020(17)$ & 0.003326 \\
8 & 109 & $2459351.622504(17)$ & 0.003332 \\
9 & -429 & $2459317.0587(13)$ & - \\
\hline
\end{tabular}

Notes. Times are given as BJD in TDB. The eclipse length could not be measured with eROSITA (data point \#9).

Higher-cadence $(1 \mathrm{~s})$ photometry was derived from the HIPPO Stokes I measurements. These revealed deep, structured eclipses, with ingress and egress showing two steps that lasted approximately $1 \mathrm{~s}$ each, indicating that the system is a two-pole accretor (see Fig. 7 for details of the observation on May 9). Eclipse timings were obtained from all four nights and are listed in Table 1.

For each eclipse, the centers of the sharp second ingress and first egress, which lasted $\sim 2 \mathrm{~s}$ each, were measured by eye. The width of the ingress and egress defined the error. The eclipse length was estimated as the time between ingress (center) and egress (center). In reality, the true eclipse length (defined as the flat bottom part of the eclipse) is $\sim 2 \mathrm{~s}$ shorter than the values shown below. The mean eclipse length was $288.0 \mathrm{~s}$, with a standard deviation of $0.4 \mathrm{~s}$. The eclipse lengths are also included in Table 1.

The time taken between the end of the eclipse of the first hot spot and beginning of the eclipse of the second is $\sim 15 \mathrm{~s}$, that is, the length of the first step at mid ingress. Likewise, the length of the egress step is $\sim 12 \mathrm{~s}$.

We also note that the eclipses are not strictly flat bottomed, implying that an additional source of luminosity is eclipsed after the white dwarf disappears, which is most likely to be the accretion stream. The depth of the eclipse, estimated from first to second contact (end of ingress), is $\sim 3 \mathrm{mag}$, while during the eclipse, the system declines by another $\sim 1$ mag at least. This implies that the minimum brightness during eclipse is
21, assuming a mean out-of-eclipse $g$ magnitude of 17 (from ASAS-SN).

A linear regression among the optical data points listed in Table 1 (first eight entries) yielded the eclipse ephemeris as given in Eq. (1),

$\mathrm{BJD}(\mathrm{TDB})=2459344.620058(9)+E \times 0.0642426(2)$.

where numbers in parentheses are the $1 \sigma$ uncertainties in the last digits. This means that with an orbital period of $\sim 92 \mathrm{~min}$, eRASSt J192932.9-560346 belongs to the short-period eclipsing objects.

The X-ray nondetection during one of the eRASS3 scans suggests that it coincided with one of the eclipses of the accreting white dwarf. This initial ephemeris was sufficiently accurate to extend back by one month and determine the cycle number with high accuracy. The corresponding time is given in the last row of Table 1 . The timing error was assumed as the eclipse half-length minus the length of the eRODAY (40 s) to stay completely within the eclipse. A linear regression among all data in the table left all values and their errors unchanged within the error margins of Eq. (1). This therefore represents our final result.

The Gaia light curve has a floor at $G \sim 20$ (see the red data points in Fig. 4), and we were tempted to associate these times with further past eclipse observations. The group of the more recent Gaia events lies 170-270 days before our time zero in Eq. (1). The accumulated timing uncertainty over an interval of 250 days is $51 \mathrm{~s} \mathrm{(0.009}$ phase units). None of the Gaia data points lies close to predicted phase zero. We conclude that the low Gaia values are not eclipse events.

\subsection{Results and conclusion}

We report the discovery of the bright strongly variable object eRASSt J192932.9-560346, which was detected in the ongoing eROSITA X-ray all-sky surveys. It was identified as a magnetic $\mathrm{CV}$, belonging to the polar subclass, through optical and X-ray spectroscopy, high-speed optical photometry, and polarimetry. While it is expected that the eRASS surveys will uncover many such objects, this object is special through its high brightness and the structured eclipse that was discovered by our initial highspeed polarimetry and photometry. The deep eclipse of $\sim 4$ mag lasts about $300 \mathrm{sec}$ and allowed us to derive a precise binary orbital period of $92.5094 \pm 0.0002 \mathrm{~min}$. The nondetection of the source during one of the eRASS3 scans was due to a coincidence with the eclipse of the accreting white dwarf.

The nondetection of eRASSt J192932.9-560346 during eRASS2 indicates that the object was in a low state at that time. Low states may occur frequently in polars, but the general properties of these states of reduced accretion (frequency, duration) are not well known. The high-to-low state transitions were studied for a few objects (Kafka \& Honeycutt 2005), and for the prototype, AM Herculis, an accretion duty cycle of $50 \%$ was derived (Hessman et al. 2000). Some polars show short low states lasting weeks, and other low states may last decades (EF Eri, AR UMa). A short low state as observed in eRASSt J192932.9-560346 during eRASS2 is therefore common.

The eclipses themselves display a very interesting two-step structure (lower panel of Fig. 7) similar to that of UZ For (Perryman et al. 2001), indicating accretion at two poles. Only a small phase offset was observed, therefore it appears likely that there is a northern and a southern pole at perhaps similar stellar longitude (observer located in the northern hemisphere). The northern pole (shorter eclipse) was found to be about $70 \%$ 
brighter in the $I$ band than the southern pole. The bright phase is centered at phase $\sim 0.75$. A pre-eclipse dip occurs and is centered at phase $\sim 0.2$, but it already begins at phase $\sim 0.77$, that is, roughly 80 degrees before eclipse center. The pre-eclipse marks the time at which our line of sight crosses the stream that feeds the northern pole. The early phase of this feature and the centroid of the optical light curves point to a somewhat unusual location of the northern (presumably the main) accretion spot approximately a quarter cycle before eclipse. The stellar longitude of the southern spot is currently uncertain, but it must lie closer to the binary meridian (line connecting both stars) because its eclipse is centered slightly earlier in phase than that of the northern region (as indicated by the different lengths of the slow ingress and egress phases between the steep ingress and egress phases)

Interestingly, eRASSt J192932.9-560346 becomes strongly positively polarized through the pre-eclipse dip. If the light from the northern spot is completely shielded at the dip, this must be synchrotron radiation from the southern spot originating at a different longitude.

Circular polarimetry shows several zero crossings, but no phase of complete absence of polarization. There must always be one of the spots in the field of view of the observer. Even during the extended phase of minimum brightness of the light curve, at about phase 0.25 , a linear polarization pulse reaching almost $15 \%$ and some positive circular polarization are found, indicating the visibility of at least one of the accretion regions.

The SALT spectra were obtained in the phase interval between $0.42-0.67$. Their blue continuum, if interpreted as high-harmonic cyclotron radiation forming a quasi-continuum, is indicative of a moderate field strength of about 20-30 MG (see, e.g., Schwope \& Beuermann 1990; Schwope et al. 1993, for cyclotron spectra of polars in this range of field strength). They expectedly do not show any sign of the secondary star. If the donor star in eRASStJ192932.9-560346 were to follow the revised sequence of Knigge (2007) for normal CVs, it would have a mass $0.108 M_{\odot}$ (spectral type about M6.5), an absolute magnitude $M_{V}=14.9$, and an apparent magnitude $V=22.8$ for the Gaia-based distance. This is too faint to be detected with our spectroscopy or fast photometry at the bottom of the eclipse.

For an estimate of the orbital inclination, we follow the method outlined by Chanan et al. (1976). For an assumed eclipse length of the white dwarf of 302 seconds and a mass ratio $M_{\mathrm{WD}} / M_{2}=0.8 / 0.108=7.4$, we derive $i=82.5^{\circ}$. For an extreme mass ratio of 10 , the maximum inclination is $84.4^{\circ}$.

eRASSt J192932.9-560346 was not detected with ROSAT, likely because it was in a low state in these days. If it had been in a high state during the RASS, it would probably still have escaped identification. It does not show the pronounced soft Xray component that led to the discovery of many polars from the RASS (e.g., Beuermann \& Schwope 1994). The soft component was regarded as a defining criterion of polars in the past, but interestingly, all the X-ray discovered polars since the RASS have not shown a spectral component like this or a soft excess (see, e.g., Schwope et al. 2020; Webb et al. 2018, for recent additions through serendipitous XMM-Newton discoveries). The new source is located at a Galactic latitude of $-27.4^{\circ}$. The Galactic Plane Survey (GPS, Motch et al. 1996) observed below $\left|b^{I I}\right|=20^{\circ}$, while the systematic follow-up of all bright sources, the ROSAT Bright Survey (RBS, Schwope et al. 2000,
2002), observed only above $\left|b^{I I}\right|=30^{\circ}$. This shows that there is a huge discovery space to be explored with the systematic follow-up of eROSITA sources that will detect objects such as eRASSt J192932.9-560346 out to a discance of 1500 pc.

Acknowledgements. This work is based on data from eROSITA, the soft instrument aboard SRG, a joint Russian-German science mission supported by the Russian Space Agency (Roskosmos), in the interests of the Russian Academy of Sciences represented by its Space Research Institute (IKI), and the Deutsches Zentrum für Luft- und Raumfahrt (DLR). The SRG spacecraft was built by Lavochkin Association (NPOL) and its subcontractors, and is operated by NPOL with support from the Max Planck Institute for Extraterrestrial Physics (MPE). The development and construction of the eROSITA X-ray instrument was led by MPE, with contributions from the Dr. Karl Remeis Observatory Bamberg and ECAP (FAU Erlangen-Nuernberg), the University of Hamburg Observatory, the Leibniz Institute for Astrophysics Potsdam (AIP), and the Institute for Astronomy and Astrophysics of the University of Tübingen, with the support of DLR and the Max Planck Society. The Argelander Institute for Astronomy of the University of Bonn and the Ludwig Maximilians Universität Munich also participated in the science preparation for eROSITA. The eROSITA data shown here were processed using the eSASS/NRTA software system developed by the German eROSITA consortium. Some of the observations presented here were obtained with SALT under the transients followup programme 2018-2-LSP-001 (PI: DB), which is supported by Poland under grant no. MNiSW DIR/WK/2016/07. D.B. and S.P. also acknowledge research support from the National Research Foundation. M.G. is supported by the EU Horizon 2020 research and innovation programme under grant agreement No 101004719.

\section{References}

Bailer-Jones, C. A. L., Rybizki, J., Fouesneau, M., Demleitner, M., \& Andrae, R. 2021, AJ, 161, 147

Beuermann, K., \& Schwope, A. D. 1994, ASP Conf. Ser., 56, 119

Beuermann, K., Thomas, H. C., \& Schwope, A. 1988, A\&A, 195, L15

Brunner, H., Liu, T., Lamer, G., et al. 2022, A\&A, 661, A1 (eROSITA EDR SI)

Buckley, D. A. H., Swart, G. P., \& Meiring, J. G. 2006, Proc. SPIE, 6267, $62670 Z$

Burgh, E. B., Nordsieck, K. H., Kobulnicky, H. A., et al. 2003, Proc. SPIE, 4841, 1463

Chanan, G. A., Middleditch, J., \& Nelson, J. E. 1976, ApJ, 208, 512

de Jong, R. S., Agertz, O., Berbel, A. A., et al. 2019, The Messenger, 175, 3

Drake, A. J., Djorgovski, S. G., Mahabal, A., et al. 2009, ApJ, 696, 870

Hessman, F. V., Gänsicke, B. T., \& Mattei, J. A. 2000, A\&A, 361, 952

Hodgkin, S. T., Harrison, D. L., Breedt, E., et al. 2021, A\&A, 652, A76

Kafka, S., \& Honeycutt, R. K. 2005, AJ, 130, 742

Knigge, C. 2007, MNRAS, 382, 1982

Kochanek, C. S., Shappee, B. J., Stanek, K. Z., et al. 2017, PASP, 129, 104502

Kollmeier, J. A., Zasowski, G., Rix, H.-W., et al. 2017, ArXiv e-prints [arXiv:1711.03234]

Merloni, A., Predehl, P., Becker, W., et al. 2012, ArXiv e-prints [arXiv:1209.3114]

Motch, C., Haberl, F., Guillout, P., et al. 1996, A\&A, 307, 459

Perryman, M. A. C., Cropper, M., Ramsay, G., et al. 2001, MNRAS, 324, 899

Potter, S. B., Buckley, D. A. H., O'Donoghue, D., et al. 2010, MNRAS, 402, 1161

Predehl, P., Andritschke, R., Arefiev, V., et al. 2021, A\&A, 647, A1

Revnivtsev, M., Sazonov, S., Churazov, E., et al. 2009, Nature, 458, 1142

Saxton, R. D., Read, A. M., Esquej, P., et al. 2008, A\&A, 480, 611

Schreiber, M. R., Belloni, D., Gänsicke, B. T., Parsons, S. G., \& Zorotovic, M. 2021, Nat. Astron., 5, 648

Schwope, A. 2012, Mem. Soc. Astron. It., 83, 844

Schwope, A. D., \& Beuermann, K. 1990, A\&A, 238, 173

Schwope, A. D., Thomas, H. C., \& Beuermann, K. 1993, A\&A, 271, L25

Schwope, A., Hasinger, G., Lehmann, I., et al. 2000, Astron. Nachr., 321,

Schwope, A. D., Brunner, H., Buckley, D., et al. 2002, A\&A, 396, 895

Schwope, A. D., Worpel, H., Webb, N. A., Koliopanos, F., \& Guillot, S. 2020, A\&A, 637, A35

Shappee, B. J., Prieto, J. L., Grupe, D., et al. 2014, ApJ, 788, 48

Sunyaev, R., Arefiev, V., Babyshkin, V., et al. 2021, A\&A, 656, A132

Warner, B. 2003, Cataclysmic Variable Stars (Cambridge: Cambridge University Press)

Webb, N. A., Schwope, A., Zolotukhin, I., Lin, D., \& Rosen, S. R. 2018, A\&A, $615, \mathrm{~A} 133$ 